\title{
Igualdad de género y conciliación familiar: entrevista con Nuria Pumar Beltrán
}

\author{
Rocio Riesco
}

Universidad de Buenos Aires, Buenos Aires, Argentina

La revisión de las políticas sociales sobre conciliación en diferentes contextos permite constatar las distancias en materia de igualdad entre sexos que existen tanto en el ámbito laboral como en el familiar. Por esta razón, la existencia de normas sobre este problema supone la toma de conciencia acerca de la necesidad de ofrecer respuestas a demandas sociales que se han generalizado en los últimos años (Marta ORTEGA GASPAR, 2012).

En ese contexto, la licencia estudiantil por maternidad y paternidad y la provisión de servicios destinados al cuidado de los/as hijos/as de estudiantes (guarderías o jardines para la primera infancia) ${ }^{1}$ no son cuestiones frecuentes en la investigación académica, a pesar de que existen jóvenes madres y padres que participan en la vida universitaria con escaso apoyo de las instituciones donde cursan sus estudios. Sin embargo, la disponibilidad de tiempo, dinero y recursos de las familias para el cuidado de niños y niñas pequeños incide directamente en la formación y en el desempeño laboral o profesional de los estudiantes/as.

En ese sentido, desde el campo del Derecho, en particular, las investigaciones en torno al cuidado destacan la importancia

\section{c) (7)}

Esta obra está sob licença Creative Commons.

'Se usan los términos "jardín maternal" y "guardería" para aludir a los servicios destinados al cuidado de la primera infancia. En diferentes países o regiones, suelen usarse denominaciones equivalentes. 
de considerar el derecho a ser cuidado, y a cuidarse, como un derecho universal para la persona, siendo necesario para su cumplimiento no sólo la promoción de una oferta de cuidado, sino también la universalización de la responsabilidad, la tarea y la asignación de los recursos materiales para realizarlo (Laura PAUTASSI, 2007-2013).

En algunos países de América Latina, en especial en la Argentina, se han promovido en los últimos años varias iniciativas orientadas a revertir algunos aspectos de esta situación de inequidad en las institucionales universitarias. En el ámbito europeo, los esfuerzos se concentraron en las llamadas "políticas de conciliación familiar", destinadas a favorecer la participación igualitaria en el mundo laboral, y, en el último tiempo, se ha promovido también la creación de Comisiones de lgualdad en el ámbito universitario.

Para conocer los avances producidos en Barcelona y, en especial, en la Facultad de Derecho de la Universidad de Barcelona, el 28 de enero de 2016 se realizó una entrevista presencial a la Dra. Nuria Pumar Beltrán. La conversación se centró en los cambios producidos en los últimos años en las políticas públicas de igualdad europeas y, en especial, en las políticas españolas y catalanas destinadas a promover la equidad de género entre los y las estudiantes universitarios. ${ }^{2}$

Nuria Pumar Beltrán dirige el Instituto Interuniversitario de Estudios de Mujeres y Género y preside la Comisión de Igualdad de la Facultad de Derecho de la Universidad de Barcelona; coordina la Clínica Jurídica Mujeres en el ámbito del Proyecto derecho al Derecho y es Profesora de Derecho del Trabajo y de la Seguridad Social en esa institución universitaria. ${ }^{3}$ También forma parte de la Red Temática Internacional de Investigación sobre Familias Monoparentales (TIIFAMO) y ha prestado especial atención al impacto familiar de las reformas laborales y de seguridad social producidas en los últimos años. Es autora de numerosas publicaciones, entre ellas, los libros Política activa de empleo y seguridad social (2003) y La igualdad ante la ley en el ámbito de la Seguridad Social (2001). ${ }^{4}$

\footnotetext{
${ }^{2}$ La entrevista es un avance parcial del proyecto "Una aproximación socio jurídica a las respuestas de las Universidades Nacionales a las necesidades de cuidado en la primera infancia. Un estudio de caso centrado en el sistema de licencias y de servicios de cuidado para los/as estudiantes universitarios", dirigido por Carla Zibecchi y financiado por una Beca Estímulo UBACyT 2015-2016. El/la autor/a integra, además, el proyecto UBACyT Interdisciplinario en Formación: "Familias y necesidades de cuidado en contextos de marginalidad social. Una mirada sociojurídica en torno a las respuestas de las políticas sociales y las sentencias judiciales", radicado en el Instituto Ambrosio L. Gioja de la Facultad de Derecho de la Universidad de Buenos Aires, desde 2014 hasta el 2017.

${ }_{3}^{3}$ Para más información ver: http://www.iiedg.org y http://www.ub.edu/dretaldret. ${ }^{4}$ Esta entrevista presencial se realizó como parte de las actividades académicas financiadas por una Beca de Movilidad del Banco Santander otorgada por la Universidad de Buenos Aires al AUTOR/A en 2015.
} 
Rocío Riesco (RR): el reparto equitativo de las tareas de cuidado de la primera infancia constituye un factor imprescindible para la búsqueda de la igualdad entre hombres y mujeres. ¿Cómo es reconocido el cuidado en el ámbito académico español? ¿Qué avances se reconocen en las últimas décadas en términos legislativos y qué discusiones se están llevando a cabo?

Nuria Beltrán Pumar (NBP): en el orden normativo, el derecho al cuidado está reconocido en la Carta de Derechos Fundamentales de la Unión Europea como derecho a la conciliación. Existe, además, una normativa europea sobre estos temas en la que el enfoque no es el del derecho al cuidado en sí, sino el del derecho a conciliar la vida laboral con la familiar, puesto que se enfatiza la creación del empleo femenino. ${ }^{5}$ La Unión Europea, en sus directrices económicas, recomienda a los Estados elevar el número de mujeres europeas que acceden a un empleo, ya que la tasa de actividad de las mujeres cambia mucho según países. En Suecia es muy alta, pero en España e Italia, no. En España, por ejemplo, es un problema también generacional. En la época de [Francisco] Franco, el contexto normativo era totalmente adverso a la participación laboral de las mujeres y, ahora, ha cambiado con las nuevas generaciones. Pero el enfoque de la conciliación está vinculado al empleo en la Unión Europea. Luego, la perspectiva que ha empezado ya a funcionar es la de corresponsabilidad, es decir, la de hacer que los hombres participen también en el cuidado. Desde esa perspectiva, entonces, la conciliación se defiende porque va muy vinculada a la igualdad de género, a la idea de los roles, y a un reparto de tareas más equilibrado. La Directiva 2010/18/UE del 8 de marzo, que sustituye a la anterior Directiva 96/34/CE del 3 de junio, habla de que entre los progenitores también la figura del padre es la que debe hacer uso del permiso parental, introduciendo la idea de la corresponsabilidad como la nueva tendencia. ${ }^{6}$ En España, la conciliación no está expresamente reconocida en la Constitución, pero sí ha habido una serie de normas laborales que han ido reconociendo una cierta flexibilidad en el uso del disfrute y la ampliación de los permisos. El permiso de maternidad ha ido evolucionando y se ha tratado de una evolución positiva. La Ley Orgánica 3/2007 del 22 de marzo, para la igualdad efectiva de mujeres y hombres, menciona que hay una "asunción colectiva de la maternidad".7 Luego, también hay que considerar las políticas que se aplican. Con la crisis, ha habido recortes importantes en materia de educación, y eso ha afectado los planes que había

\footnotetext{
${ }^{5}$ El artículo 11 de la Resolución del Parlamento Europeo, de 27 de septiembre de 2007, sobre la igualdad entre mujeres y hombres en la Unión Europea - 2007 (2007/2065(INI)) demuestra la relación entre derecho al cuidado y empleo: "Pide a los Estados miembros que introduzcan medidas específicas para eliminar las desigualdades entre mujeres y hombres derivadas de la interrupción del empleo, sobre todo debido a una maternidad o a la necesidad de atender a personas dependientes, así como para reducir sus efectos negativos sobre la carrera laboral, las retribuciones y los derechos de pensión y que trabajen en pro de unos salarios y unas pensiones no sexistas; pide a la Comisión que encuentre medios apropiados para combatir la segregación por razón de género en el mercado laboral y para facilitar la incorporación de las mujeres a los sectores no tradicionales;". Un detalle más amplio de la normativa vigente sobre este problema en Catalunya, en España y en la Unión Europea, puede consultarse en: http://www.ub.edu/web/ub/ca/sites/genere/normativa/normativa.html ${ }^{6}$ Un detalle de los avances producidos sobre el derecho al cuidado en el mundo laboral europeo puede verse en ORTEGA GASPAR, 2012.

${ }^{7}$ Véase el apartado 7 del Artículo 14 donde, dentro de las políticas públicas para la igualdad, se menciona como criterio general de actuación de los Poderes Públicos "la protección de la maternidad, con especial atención a la asunción por la sociedad de los efectos derivados del embarazo, parto y lactancia". En el mismo sentido, ver el Artículo 44: "Los derechos de conciliación de la vida personal, familiar y laboral. 1. Los derechos de conciliación de la vida personal, familiar y laboral se reconocerán a los trabajadores y las trabajadoras en forma que fomenten la asunción equilibrada de las responsabilidades familiares, evitando toda discriminación basada en su ejercicio". (Comp.) Las fronteras del cuidado. Agenda, derechos e infraestructura. Buenos Aires: Editorial Biblos, 2013, pp. 99-132.
} 
respecto a guarderías. ${ }^{8}$ El permiso de paternidad se pretendía ampliar de dos a cuatro semanas y esta intención se ha ido posponiendo. Y con la crisis económica, las políticas de conciliación no se priorizan, porque pueden suponer un coste económico.

RR: en el marco de la Unión Europea, las políticas de conciliación conforman uno de los aspectos que contribuyen a la búsqueda de la igualdad entre hombres y mujeres, apoyando directamente la combinación de la vida profesional y la vida privada. ¿Qué políticas se incluyen en el marco de las políticas de conciliación en el ámbito universitario y cuáles están dirigidas, en especial, a los y las estudiantes?

NPB: aquí, en Barcelona, desconozco una figura de políticas de conciliación para estudiantes/as universitarios/as, aunque no me especializo en ese tema. La Ley Orgánica de Igualdad obliga a todas las empresas tanto públicas como privadas, que sean de una determinada dimensión (plantilla de más de 250 trabajadores), a tener un Plan de lgualdad, que diagnostiquen cuáles son los problemas que hay. Casi todos los Planes de Igualdad contienen un capítulo dedicado a la conciliación del personal, pero es muy distinta la normativa que se aplica en la universidad al personal de administración y servicios (PAS), que la que se aplica al profesorado. Hay muchas figuras jurídicas y sería bastante complejo ahora resumirlo, pero la realidad es que estos Planes de lgualdad normalmente ignoran la figura del estudiante. En España ha habido un cierto "envejecimiento"; la maternidad y paternidad se han ido retrasando tanto que, en general, las estudiantes en situación de embarazo son pocas, y la mayoría de los casos se da en estudiantes que no han seguido la carrera habitual de estudios, sino que han trabajado y luego han estudiado. Creo que no hay ninguna previsión específica en la universidad. El permiso de maternidad, tal como está concebido, tiene, en la legislación, un enfoque hacia el ámbito laboral. Es bastante universal, porque cubre a todo tipo de trabajadoras; las trabajadoras autónomas también tienen derecho no sólo al descanso, sino a una prestación económica, pero ese descanso sólo se piensa en sentido laboral, no ha sido trasladado al estudiante. La estudiante que está embarazada, que tiene un hijo en la carrera, sólo va a tener una respuesta si es trabajadora, pero a nivel de estudios, no. Por tanto, si hubiese un reconocimiento más universal del permiso de maternidad, se tendrían que ver otras facetas de la mujer, como es, por ejemplo, el ámbito del estudio. El permiso de maternidad se ha implementado en otros niveles; por ejemplo, se está intentando llevarlo a cabo en el profesorado universitario para que los períodos de maternidad no penalicen la valoración de la actividad de investigación de las mujeres. Ahora bien, en relación a los estudiantes, partimos de una especie de anomia normativa. Las universidades deberían trabajar en el problema. Creo que hay dos factores esenciales; por un lado, el retraso de la maternidad generalizado, en la medida en que la maternidad en España es de las más tardías de la Unión Europea y que la tasa de natalidad es muy baja; y, por el otro, una distinción entre clases sociales. Ahora, hay un marco normativo bastante protector del embarazo en el trabajo; la licencia por maternidad es bastante universal, en la medida en que toda mujer que trabaja va a tener derecho y la protección es del $100 \%$ del salario que estaba cobrando, pero, en el ámbito de la universidad, todo lo que no se entiende como trabajo, entre ello, el estudio, no se encuentra cubierto. Por ejemplo, si una estudiante está en situación de embarazo, no tiene ninguna tutoría especial, ni se le puede ofrecer un paréntesis, y ello tendría que servir para repensar

\footnotetext{
${ }^{8}$ La entrevistada alude a la crisis económica visible en España desde los últimos años de la década pasada. Un mayor detalle acerca de la relación entre la crisis económica española y las políticas de conciliación puede verse en Inés CAMPILLO POZA, 2014
}

4 Revista Estudos Feministas, Florianópolis, 26(1): e44559 
no sólo la maternidad, sino también otro tipo de bajas como, por ejemplo, las médicas. Creo que, en ese sentido, no se favorece la natalidad en ningún estamento y, en la universidad, aún menos.

RR: ¿Cuándo y por qué se crea la Unidad de Igualdad en la Universidad de Barcelona? ¿Cuáles son sus principales objetivos? ¿Cuáles han sido los logros más destacados y las políticas que aún quedan pendientes?

NPB: las Unidades de Igualdad están amparadas en la Ley Orgánica de lgualdad. Se han ido creando según cada universidad. El boom se dio a partir de la Ley Orgánica que exigía planes de igualdad a las empresas, no Unidades de Igualdad. ${ }^{9} Y$ se han ido creando, se coordinan, tienen reuniones anuales a nivel estatal, y en cada universidad tienen un encaje distinto dentro de lo que es el organigrama; por ejemplo, aquí, en la Universidad de Barcelona, la responsable de la Unidad de Igualdad depende del rector, es una delegada del rector y, en otros casos, están incorporadas en otros tipos de vicerrectorado. ${ }^{10}$ El problema principal que tienen las Unidades de Igualdad es conseguir suficiente apoyo administrativo para realizar todo; es decir, el Plan de Igualdad es bueno como documento porque se plantean distintos ejes y estrategias prioritarias; por ejemplo, se fijan objetivos tales como que la docencia esté libre de estereotipos, pero el problema es la falta de medios, de apoyo técnico, y eso es desigual; depende de la universidad. ${ }^{11}$ En la Universidad de Barcelona, ha sido un buen empuje el hecho de que la universidad esté en una liga llamada LERU (League of European Research Universities). Es una liga de universidades de investigación intensiva, dentro de la cual se crean grupos de trabajo entre las distintas universidades que son influyentes en el plan europeo. En mi caso, integro el Grupo de Trabajo sobre Género, donde se comparan las políticas de UB con las de otras universidades. No se trata tanto el problema estudiantil sino que se inclina más hacia la faceta de investigación y se comparten indicadores comunes de desigualdad. Un ejemplo es el escaso porcentaje de mujeres catedráticas en relación a los hombres (19\% de catedráticas de media en todas las universidades integrantes de la liga). En este sentido, la UB se halla ligeramente por encima de la media (el $22 \%$ de las cátedras son ocupadas por mujeres), si bien la cifra continúa siendo claramente insuficiente y conviene hacer un seguimiento de estos datos en el tiempo. Ahora bien, por ejemplo, respecto a la dotación presupuestaria destinada a las políticas de igualdad, la UB se situaría en los últimos puestos en relación con los presupuestos de otras universidades LERU. Cuando se entra en la Unidad de Igualdad hay tantos aspectos por trabajar, que realmente se ha empezado por unos y descuidado otros. Las políticas de conciliación son uno de los aspectos que, quizá, queda más en segundo plano, porque se solapa con otro tipo de políticas. Es un problema que falta desarrollar dentro de la Unidad, ya que está más centrada en violencia de género y en los protocolos de acoso, en cómo introducir el tema de violencia, la igualdad y la erradicación de los estereotipos en la docencia y la investigación, ya que una de las tendencias europeas es que los proyectos de investigación tienen que introducir la

\footnotetext{
${ }^{9}$ Los planes de igualdad en las empresas fueron introducidos en España por la Ley Orgánica 3/2007 para la igualdad efectiva de mujeres y hombres (LOIEMH) como instrumentos para alcanzar su igualdad material en el ámbito laboral. 10 Para una descripción general de la Unidad de Igualdad de la Universidad de Barcelona y de sus objetivos y actividades, véase: http://www.ub.edu/web/ub/ca/sites/genere/index.html.

${ }^{11}$ El Plan de Igualdad de oportunidades entre las mujeres y los hombres en la Universidad de Barcelona puede consultarse en: http://www.ub.edu/web/ub/ca/sites/genere/pla_igualtat/pla_igualtat.html.

12 Véase Nuria PUMAR BELTRÁN, 2015.
} 
perspectiva de la mujer y del género. ${ }^{12}$ En la universidad, parece que la organización y el mantenimiento de guarderías para niñas y niños fuera un asunto del Ayuntamiento, ya que los permisos por maternidad y paternidad ya existen en la legislación estatal. Asimismo, por ejemplo, en la Comisión de Igualdad de la Facultad se cuenta con estudiantes, pero a la hora de estar presentes en la Unidad, rara vez lo están. Otro motivo para entender por qué no hay este tipo de políticas para estudiantes es porque falta motivar, y eso es una autocrítica. Además, la idea de los estudiantes se tiene que ampliar también a tercer ciclo, ${ }^{13}$ porque, normalmente, la visión del estudiante es la del estudiante de grado, pero también tendría que ser la del doctorado, del máster, en la medida en que son, además, los que podrían plantear, sobre todo por su edad, alguna cuestión sobre la maternidad o paternidad.

RR: en la Unión Europea existen normativas que regulan los derechos de las y los estudiantes universitarios que deben ejercer tareas de cuidado, incluyendo embarazos, aquellos cuyas parejas son padres, o bien en casos de adopción. En el Reino Unido, universidades como Oxford, Cambridge, Nottingham, Leeds, Birmingham cuentan con estos sistemas. ¿Cree posible que España y, en especial Catalunya, avance hacia una implementación de normativas universitarias que garanticen una igualdad de derechos para hombres y mujeres a la hora de afrontar las tareas de cuidado?

NPB: eso puede ser muy interesante. Está claro que son políticas en las cuales se incluye la perspectiva del derecho al cuidado, y aquí realmente no las hay, porque la maternidad se piensa que es un asunto que debe asumir la mujer, ella sola en su circunstancia. Hay situaciones que merecen especial atención, y la de las estudiantes embarazadas es una. Ello debería ser ampliable a situaciones de permiso, paternidad y, por sobre todo, al embarazo, y tener en cuenta la situación de riesgo de la salud que puedan tener. Por ejemplo, aquí en la Facultad de Derecho ha habido una cierta dilación ante el tema de la accesibilidad de la discapacidad, que ha tardado mucho, pero en otras facultades, donde hay laboratorios, donde tienen que trabajar con tóxicos, etc., el problema está más desarrollado. En casi todas las universidades hay una unidad de riesgos laborales y se aplica en forma indistinta. Pero falta implementarlo mejor. La visión de protección global está muy enfocada al trabajo, a facilitar el empleo, pero a veces se deja en un segundo plano el derecho al cuidado y luego, también, el tema de la igualdad de género, de favorecer que el padre pueda acceder también a ciertos derechos. Es probable que España avance en ese sentido por el hecho de estar en la Unión Europea; hay una necesidad de ejercer buenas prácticas y el derecho europeo tiene mucha importancia en este sentido. Es muy probable que, sobre todo si es una experiencia anglosajona, pueda ofrecer un punto de partida. Vamos evolucionando. Una situación diferente se da en el caso de las políticas sociales. Si bien en la Unión Europea se habla mucho de conciliación, cuando se tiene que aportar dinero para hacer guarderías, no se hacen. En la UB no contamos con la figura de la guardería tampoco. Se ha planteado más de una vez, se comentó, se dijo que era un plan muy caro, y, por otro lado, hay una perspectiva sindical que no es favorable a tener la guardería, o en catalán diríamos "el hogar de los infantes". No sólo se dijo que era caro, sino que el planteamiento era que el hijo esté en un lugar próximo al lugar en el que vive y no al del lugar de trabajo. Es una situación que tiene dos caras, una situación ideológica que se puede entender desde la perspectiva del niño, pero desde la perspectiva de los padres, no tanto. Hay que pensar que esta universidad es enorme, hablamos cerca de

${ }^{13}$ La categoría "tercer ciclo" equivale a "posgrado".

14 "Instituto" equivale a la educación en el nivel secundario.

6 Revista Estudos Feministas, Florianópolis, 26(1): e44559 
cuarenta mil personas, y en ninguna facultad se cuenta con una guardería ni para estudiantes, ni para profesorado.

RR: ¿Reconoce impactos diferenciales en las trayectorias académicas de los/las estudiantes que han tenido que ejercer tareas de cuidado durante sus estudios?

NPB: El problema es que los casos que llegan a nuestro conocimiento son de mujeres que no sólo tienen hijos, sino que también trabajan, estudian, todo ello con un gran desgaste, y, si bien parece una generalización, creo que las mujeres que se atreven a dar este paso son muy especiales, en el sentido de que son muy exigentes, suelen ser de una determinada edad, ya que madres de 18 a 23 años no se encuentran habitualmente en la universidad. Sería interesante también trasladar la pregunta a los embarazos en el Instituto, ${ }^{14}$ donde la familia juega un papel clave. Si bien pueden haber cambiado las relaciones familiares, hay mucha más diversidad en familias también, pero el vínculo familiar, aún en el ámbito universitario, sigue siendo muy importante. Es la línea de ser una sociedad muy familiarista, un Estado de Bienestar que confía en las redes familiares. En España el problema lo tienen principalmente muchas mujeres inmigrantes que no tienen esas redes familiares, y se enfrentan a un verdadero problema de conciliación entre tener hijos y estudiar o trabajar.

RR: ¿Considera que el acceso a licencias y sistemas de cuidado para estudiantes universitarios/as puede contribuir en materia de igualdad?

NPB: La conclusión sería que no hay políticas de conciliación dirigidas a los estudiantes y habría que ver las razones de esta carencia legislativa. Esta falta da pie a que sea trabajada y a observar que allí hay una necesidad. En este país es una necesidad, pero, quizá, cuantitativamente, en este momento, no se le da una prioridad, porque, en el fondo, la idea de circunscribir la maternidad a la familia y a la mujer es una filosofía difícil de modificar.

\section{Referencias}

ASTELARRA, Judith. Veinte años de Políticas de Igualdad. Valencia: Cátedra, 2005.

CAMPILLO POZA, Inés. "Desarrollo y crisis de las políticas de conciliación de la vida laboral y familiar en España (1997-2014). Un marco explicativo". Investigaciones Feministas, vol. 5, pp. 207-231, 2014.

CARRASCO, Cristina, BORDERÍAS, Cristina, y TORNS, Teresa. "El trabajo de cuidados: antecedentes históricos y debates actuales". En: CARRASCO, Cristina, BORDERÍAS, Cristina y TORNS, Teresa (Eds.). El trabajo de cuidados. Historia, teoría y políticas. Madrid: Catarata, 2011.

CARRASQUER OTO, Pilar, MASSÓ, Matilde y ARTILES, Antonio M. "Discursos y estrategias en torno a la conciliación de la vida laboral y familiar en la negociación colectiva". Papers: revista de sociología, no. 83, p. 13-36, 2007.

ORTEGA GASPAR, Marta. "Evolución de las políticas sociales de conciliación en Europa". Revista de Responsabilidad Social de la Empresa, n. 11, pp. 45-76, 2012.

PAUTASSI, Laura. El cuidado como cuestión social: un aproximación desde el enfoque de derechos, Serie Mujer y Desarrollo No 87. Santiago de Chile: CEPAL, 2007.

PAUTASSI, Laura. "Perspectivas actuales en torno al enfoque de derechos y cuidado. La autonomía en tensión". En: PAUTASSI, Laura y ZIBECCHI, Carla (Coord.) Las fronteras del cuidado: agenda, derechos e infraestructura. Buenos Aires: Equipo Latinoamericano de Justicia y Género (ELA) y Biblos, 2013. p. 99-132. 
PUMAR BELTRÁN, Nuria. “Los protocolos de actuación frente al acoso: dónde existen, dónde operan y dónde son eficaces. Ejemplos de derecho comparado". En: CABALLERO PÉREZ, María José (Coord.), TOMÁS JIMÉNEZ, Natalia (Coord.), RIVAS VALLEJO, María Pilar (Dir.) y GARCÍA VALVERDE, María Dolores (Dir.). Tratamiento integral del acoso. Cizur Menor, Navarra: Thomson Reuters-Aranzadi, 2015, pp. 269-293.

[Recebida em 10/05/2016 e aprovada em 15/02/2017]

Rocio Riesco (rocio_riesco@hotmail.com). Nacida en 1992 en General Pico, La Pampa, Argentina. Estudiante de Abogacía en la Universidad de Buenos Aires, actualmente cursando el último año de la carrera. Integrante de un proyecto de investigación en la misma universidad, cuyo título es "Familias y necesidades de cuidado en contextos de marginalidad social. Una mirada sociojurídica en torno a las respuestas de las políticas sociales y las sentencias judiciales". Miembro del equipo de Derechos Sociales y Políticas Públicas (www.dspp.com.ar).

8 Revista Estudos Feministas, Florianópolis, 26(1): e44559 